\title{
A Method to Construct Volcano Relations by Multi-Scale Modeling: Building Bridges Between the Catalysis and Biosimulation Communities
}

\author{
Kai S. Exner, ${ }^{1,2, *}$ Anela Ivanova ${ }^{1}$ \\ ${ }^{1}$ Sofia University, Faculty of Chemistry and Pharmacy, Department of Physical Chemistry, \\ 1 James Bourchier Ave., 1164 Sofia, Bulgaria \\ ${ }^{2}$ Cluster of Excellence RESOLV, Bochum, Germany
}

* Corresponding author: E-mail: kai.exner@alumni.uni-ulm.de
ORCID: 0000-0003-2934-6075 (KSE); 0000-0001-6220-7961 (AI)

Supporting Information (SI)

\section{Investigated model NP-DBP-DOX}

The investigated drug-delivery system (DDS) component consists of a methyl thiolate-protected gold nanoparticle $(\mathrm{Au}-\mathrm{NP})^{[\mathrm{S} 1]}$ as carrier moiety that is linked by a covalent $\mathrm{Au}-\mathrm{S}$ bond of a terminal cysteine residue to a specially designed drug-binding peptide (DBP). ${ }^{[\mathbf{S 2}, \mathbf{S 3}]}$ The atomic coordinates of the Au-NP applied in this study are taken from the literature. ${ }^{[\mathbf{S 1}]}$ The Au-NP consists of $144 \mathrm{Au}$ atoms, from which 114 atoms form an icosahedral core while the other 30 atoms are located at the surface. All $30 \mathrm{Au}$ surface atoms are capped by two methyl thiolate groups each. Further details can be found in our recent publication. ${ }^{[\mathbf{S} 4]}$

The peptide consists of 13 amino acid residues with the following sequence: $\mathrm{NH}_{3}{ }^{+}-$ CGGLWSPWYGGSW-CONH 2 , in which the amino group of the N-terminus is positively charged at physiological conditions. ${ }^{[\mathbf{5 5}, \mathbf{S 6}]}$ This DBP was shown to be a suitable carrier for the drug doxorubicin (DOX) due to the presence of several tryptophan residues that are able to stack or intercalate DOX. ${ }^{[\mathbf{5 7}, \mathbf{8 8}]}$ The cytostatic DOX belongs to the class of anthracycline antibiotics, consisting of an anthracycline body, a sugar residue and a short side chain. The sugar entity of the drug is positively charged at physiological conditions. All positive charges in the simulations are neutralized by chloride anions.

It should be noted that the particular chemical composition of the studied DDS component is adopted from an experimental study of Peneva and coworkers based on the previous publications ${ }^{[\mathbf{S 2}, \mathbf{S 3}]}$ to render it synthetically feasible. Figure S1 summarizes the composition of the NP-DBP-DOX associate.

\section{Computational details}

We performed atomistic molecular dynamics (MD) simulations for the three-body DDS component of Figure S1, thereby analyzing two independent trajectories generated with different initial velocities. The associate NP-DBP-DOX is solvated in an orthorhombic box with edge sizes of $(6 \times 6 \times 7) \mathrm{nm}$, applying periodic boundary conditions. Sodium and chloride ions are added to mimic physiological conditions of the human body at $T=310 \mathrm{~K}$; that is, the $\mathrm{NaCl}$ concentration is $154 \mathrm{mmol} / \mathrm{L}$. The number of explicit water molecules in the simulations is 7759 . The overall simulation time amounts to $300 \mathrm{~ns}$ and $200 \mathrm{~ns}$ for trajectories 1 (TRAJ 1) and 2 (TRAJ 2), respectively. 
The force field AMBER03 ${ }^{[\mathrm{S9}]}$ is used for DOX, DBP, as well as for the sodium cations, chloride anions, and the caps of Au-NP. The solvent is modeled by TIP3P. ${ }^{[\mathbf{S 1 0 , 5 1 1 ]}}$ Parameters used for the drug and the DBP are specified in previous publications. ${ }^{[\mathbf{S 7 , S 8 , S 1 2 ]}}$ The parameters for the covalent $\mathrm{Au}-\mathrm{S}$ junction between Au-NP and DBP as well as non-bonded parameters for Au are taken from the study of Giri and Spohr. ${ }^{[\mathbf{S 1 3}]}$ RESP charges ${ }^{[\mathbf{S 1 4}]}$ are derived for the $\mathrm{Au}, \mathrm{C}, \mathrm{S}$, and $\mathrm{H}$ atoms of the caps. An overview is given in Table S1.

\section{a) DDS constituents}
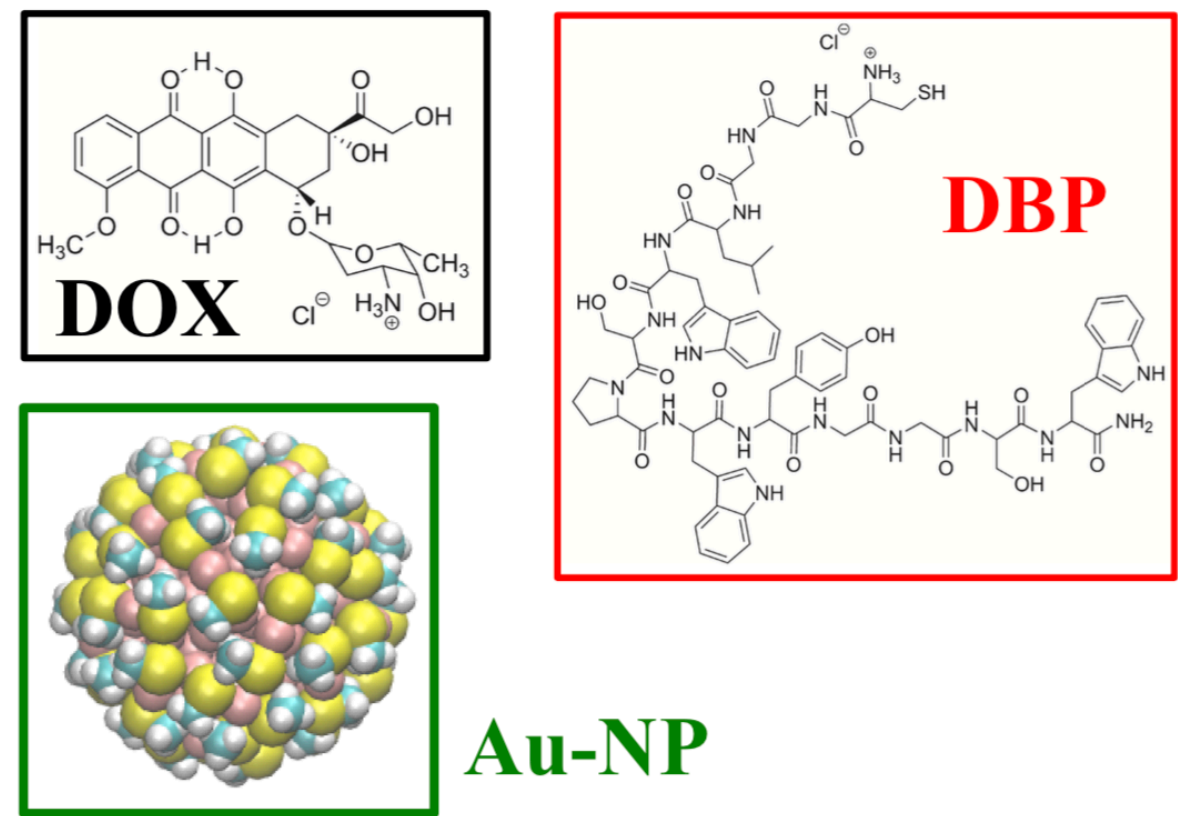

\section{Au-NP}

b) MD

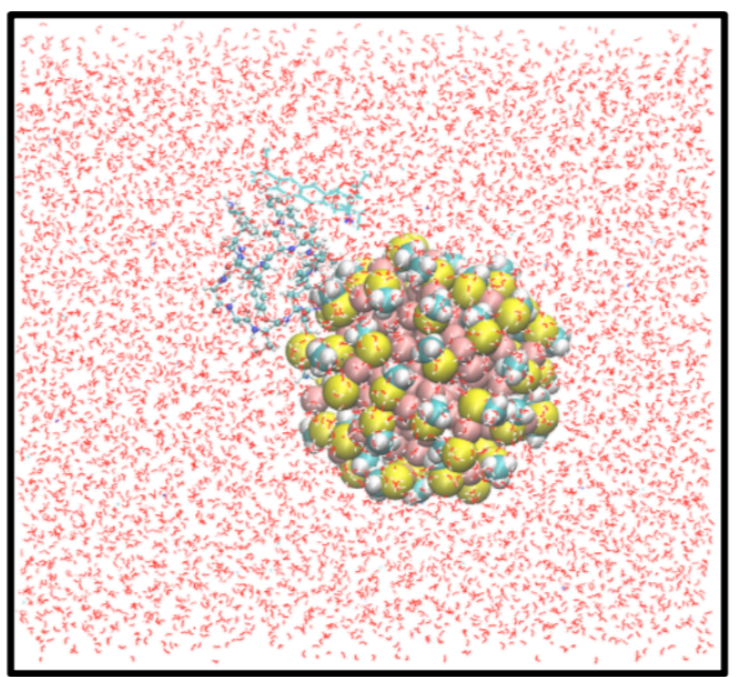

c) DFT

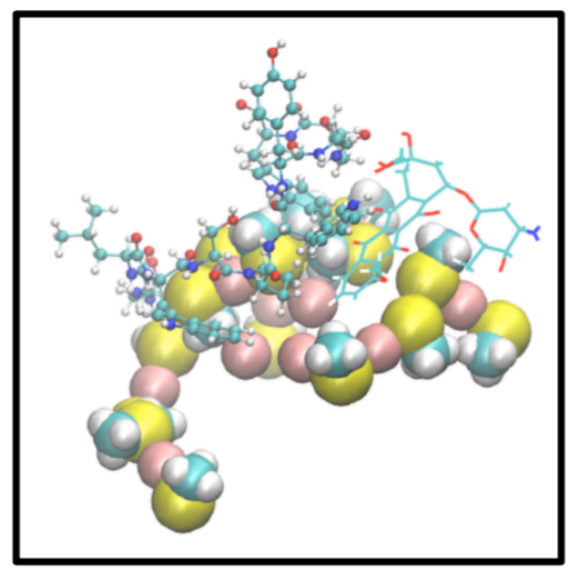

Figure S1. a) Components of the studied model drug-delivery system (DDS): the drug doxorubicin (DOX) and a drug-binding peptide (DBP) as well as a methyl-thiolate-protected gold nanoparticle (Au-NP) as carrier moieties. b) Solvated model of the NP-DBP-DOX associate in an orthorhombic box for the atomistic molecular dynamics (MD) simulations. c) Model of the NPDBP-DOX conjugate, consisting of 351 atoms, for the density functional theory calculations (DFT). All parts of the Au-NP that do not interact directly with DBP-DOX are cut out for computational feasibility. The Au-NP is sketched with vdW spheres and lines are used for DOX. Amino acids of the DBP are in CPK representation. 
Table S1. RESP charges of cap atoms employed in the MD simulations.

\begin{tabular}{|c|c|}
\hline Atom & Charge \\
\hline $\mathrm{Au}$ & +0.172 \\
$\mathrm{C}$ & -0.176 \\
$\mathrm{~S}$ & -0.216 \\
$\mathrm{H}$ & +0.102 \\
\hline
\end{tabular}

For all $\mathrm{Au}$ atoms of the Au-NP, position restraints with a force constant of $1800 \mathrm{~kJ} /\left(\mathrm{mol}_{\mathrm{nm}}{ }^{2}\right)$ are imposed during all MD simulations to prevent the Au-NP from dissociating, discussed in more detail in our recent work. ${ }^{[\mathbf{S 4}]}$ We employ Berendsen barostat ${ }^{[\mathbf{S 1 5}]}$ and $\mathrm{v}$-rescale thermostat ${ }^{[\mathbf{S 1 6}]}$ to maintain conditions close to those in vivo, i.e., constant pressure of 1 bar and temperature of $310 \mathrm{~K}$. During the simulations, all hydrogen-containing bonds are restrained by $\operatorname{LINCS}^{[\mathbf{5 1 7 ]}}$ or SETTLE ${ }^{[\mathbf{S 1 8}]}$ for the organic molecules and water, respectively. A Lennard-Jones potential with a cutoff of $1.2 \mathrm{~nm}$ and a switch function activated at $1.0 \mathrm{~nm}$ describes the non-bonded interactions, while electrostatics is assessed in the monopole approximation with the PME method, ${ }^{[\mathbf{S 1 9 ]}}$ applying a cutoff of $1.2 \mathrm{~nm}$ and a switch function turned on at $1.0 \mathrm{~nm}$. The 1-BFGS algorithm ${ }^{[\mathbf{S 2 0}, \mathbf{S 2 1}]}$ is used for initial energy minimization, while the integration of the equations of motion is performed with leap-frog, ${ }^{\left[{ }^{[22}\right]}$ using a time step of 2 fs. Snapshots are saved in the trajectories every 0.2 ps or 2 ps for TRAJ 1 and TRAJ 2 , respectively.

All MD simulations are carried out following a standard protocol: in the first step, energy minimization with position restraints of $1000 \mathrm{~kJ} /\left(\mathrm{mol} . \mathrm{nm}^{2}\right)$ on all non-hydrogen atoms is performed. The second step is an unrestrained energy minimization (except for the Au atoms within the Au-NP, for which the restraints are always kept). After that the system is heated to $310 \mathrm{~K}$ at a constant rate of $0.002 \mathrm{~K} / \mathrm{step}$, followed by a relaxation phase in NPT ensemble. While TRAJ 1 equilibrates within 50 ns, $75 \mathrm{~ns}$ are required for TRAJ 2. The equilibration is verified by the evolution of the total energy, temperature, pressure, and the root-mean-square deviation (RMSD) of the atomic coordinates of the highly flexible DBP as well as of DOX with respect to the coordinates of the minimized structure. Figures S2, S3, and S4 illustrate the equilibration process for both trajectories. Thereafter, data collection takes place within a production phase: only the remaining part of the production trajectories ( $250 \mathrm{~ns}$ and $125 \mathrm{~ns}$ for TRAJ 1 and TRAJ 2, respectively) is used for statistical analysis. All MD simulations are carried out with the software package GROMACS 5.1.2. ${ }^{[\mathbf{S 2 3}]}$ VMD 1.8.6 $6^{[\mathbf{S 2 4}]}$ is used for visualization.

Cluster analysis for the two production trajectories of the associate NP-DBP-DOX is performed with the method of Jarvis-Patrick, ${ }^{[\mathbf{S 2 5}]}$ in which a cutoff distance of $0.115 \mathrm{~nm}$ is used. Clusters with a relative population of at least $3 \%$ are considered as representative of a given structural type, referring to previous

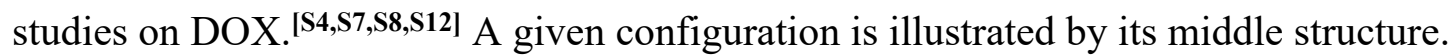

For the middle structures of all representative clusters, a model consisting of 351 atoms (all 69 atoms of DOX, all 192 atoms of DBP, 90 atoms from the capped Au-NP, Figure S1) is subject to DFT calculations. $^{[\mathbf{S 4 ]}}$ The parts of the Au-NP that do not interact directly with DBP-DOX are cut out for computational feasibility. In order to obtain the binding energies (BEs) between the three parts of the DDS component, DFT with dispersion correction is employed, utilizing B3LYP-D3 as functional ${ }^{[\mathbf{S 2 6}}$ and 6-31G* as basis set for all atoms except $\mathrm{Au}$, for which LANL2DZ ${ }^{[\mathbf{5 2 7}]}$ is applied. The geometry of the model as obtained in the MD simulations is maintained and only single point calculations with DFT are carried out. The BEs are calculated for the dimers DOX-DBP, DOX-NP, and DBP-NP in vacuo. Basis set superposition error (BSSE) counterpoise correction ${ }^{[\mathbf{S 2 8}]}$ is done in order to alleviate overbinding effects. All DFT calculations are performed with GAUSSIAN16. ${ }^{\text {[S29] }}$ 
a)

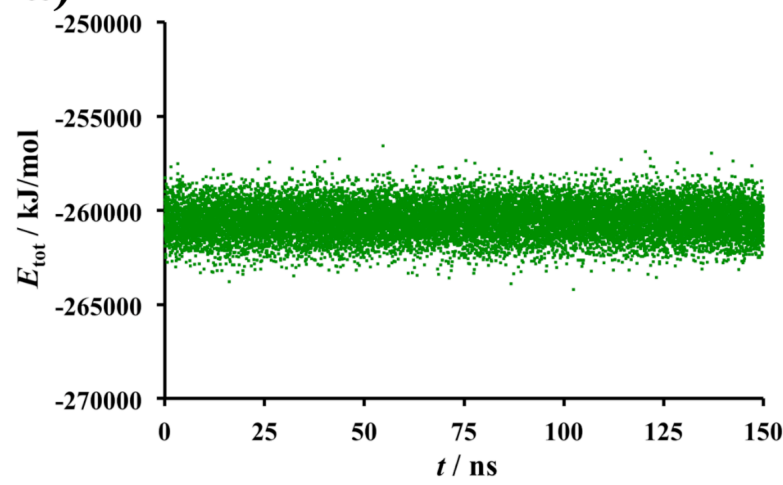

c)

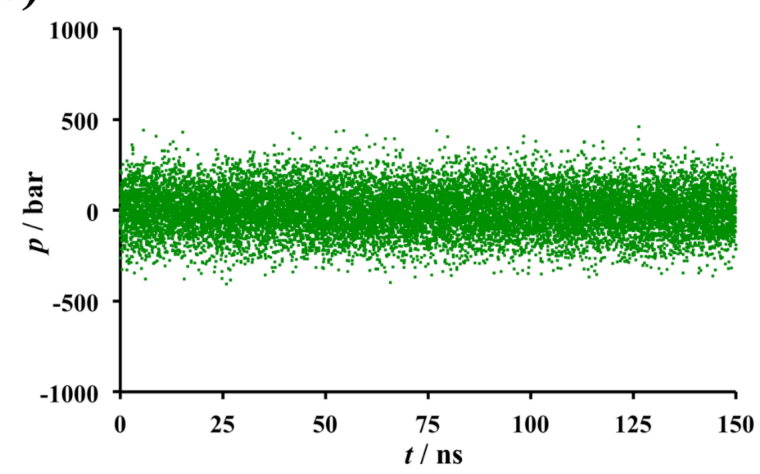

b)

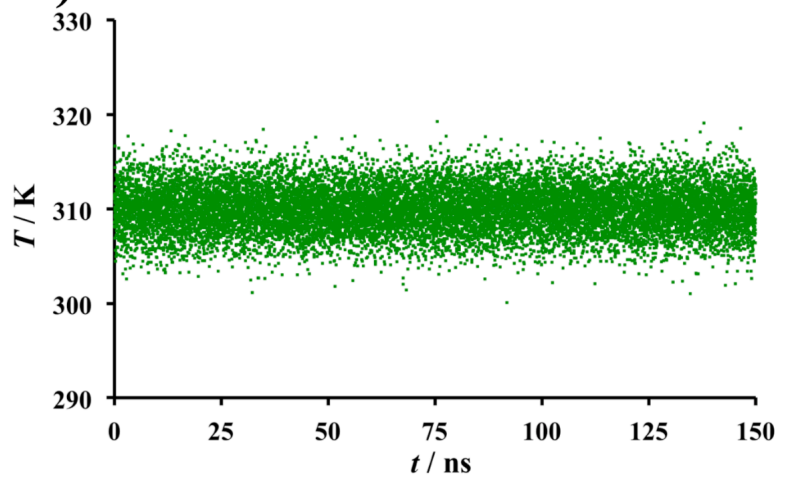

Figure S2. a) Total energy, b) temperature, and c) pressure of the system as a function of time for NP-DBP-DOX during the production phase of the first trajectory (TRAJ 1). The first $150 \mathrm{~ns}$ of the production phase are depicted in the respective panels to allow comparison with the production phase of the second trajectory, shown in Figure S3.
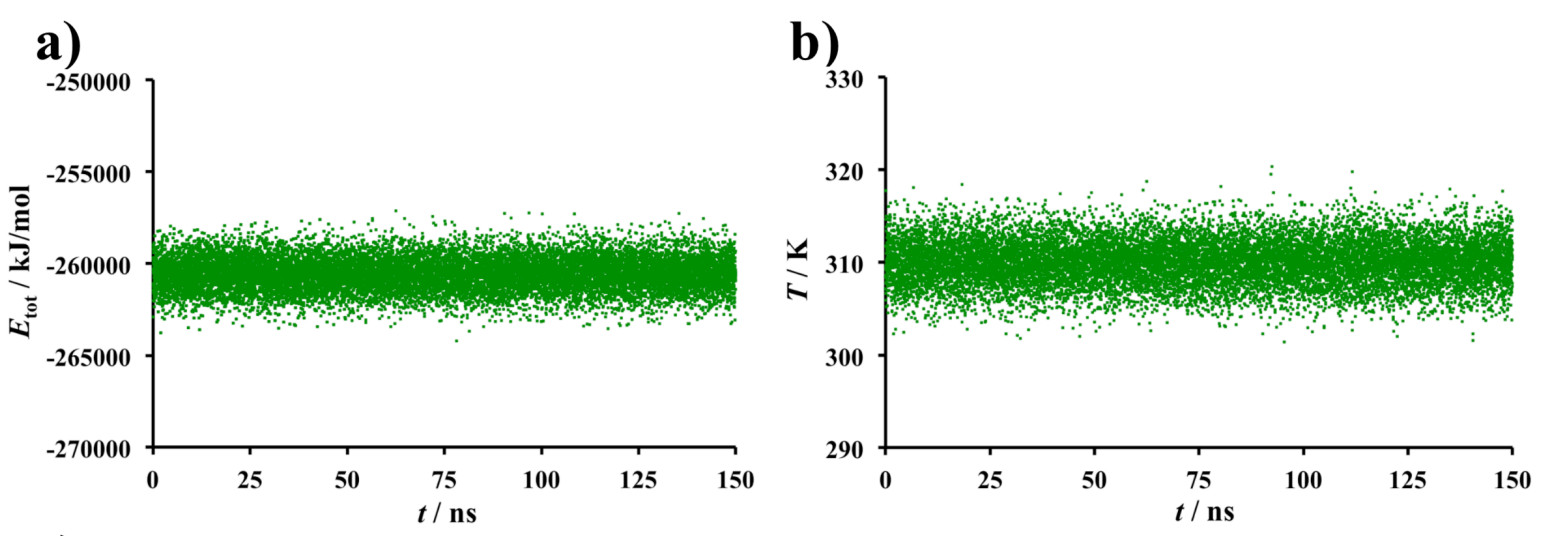

c)

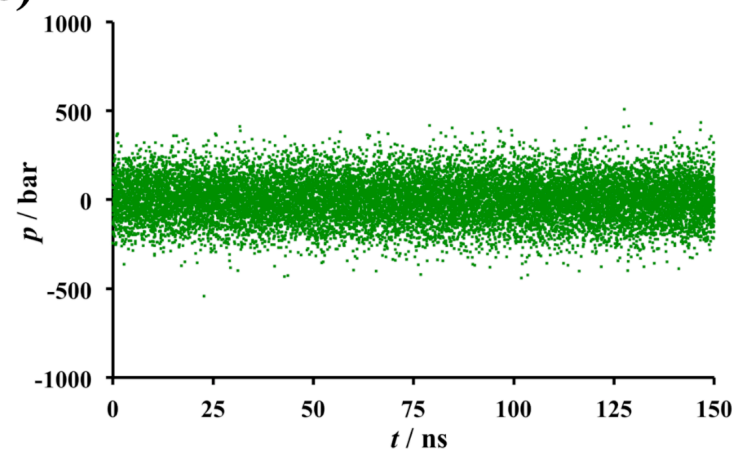

Figure S3. a) Total energy, b) temperature and c) pressure of the system as a function of time for NP-DBP-DOX during the second trajectory (TRAJ 2). The last $25 \mathrm{~ns}$ of the equilibration phase and the entire $125 \mathrm{~ns}$ of the production phase are shown to allow comparison with Figure S2. 
a)

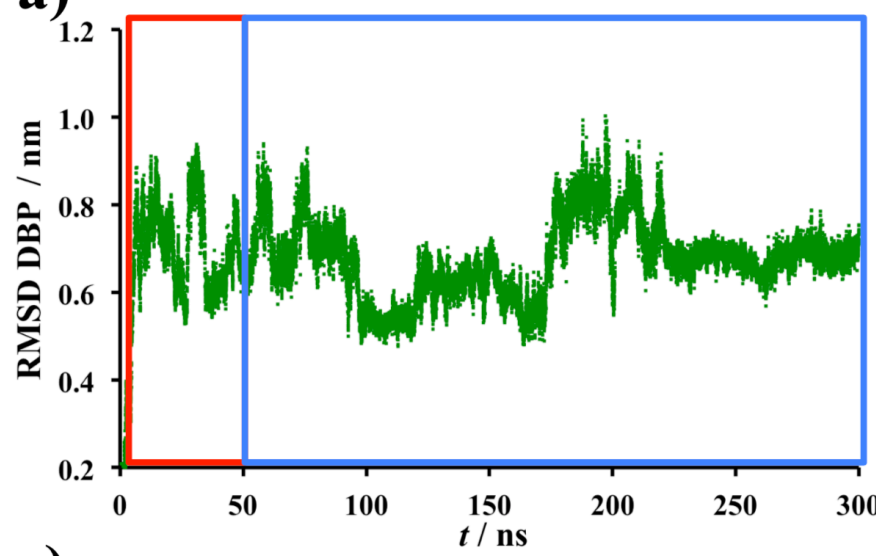

c)

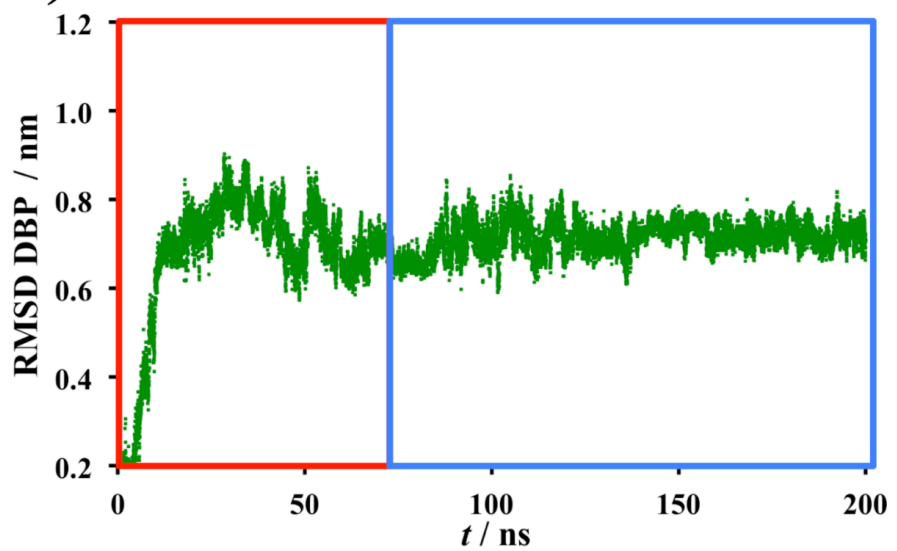

b)

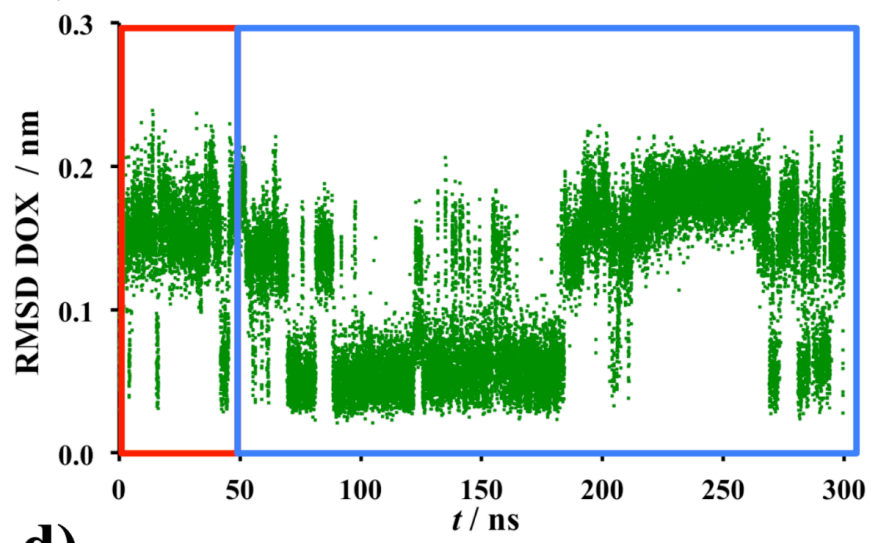

d)

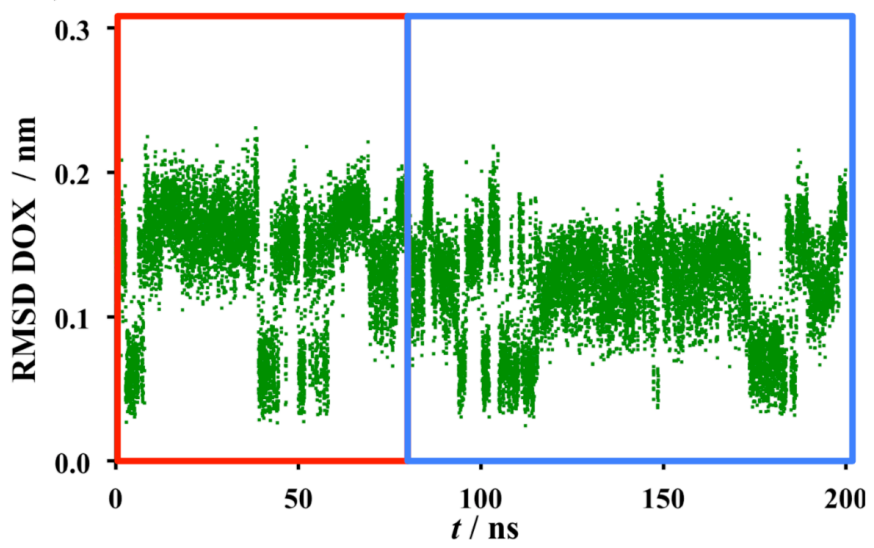

Figure S4. RMSD of a) and c) DBP and b) and d) DOX in the associate NP-DBP-DOX during the equilibration and production phases of (top row) TRAJ 1 and (bottom row) TRAJ 2; all RMSD plots use the initial energy-minimized structure as reference. The respective equilibration and production phases are marked by red and blue boxes, respectively.

\section{Trajectory analysis}

A detailed analysis of the physical properties of the Au-NP, after linking the DBP-DOX conjugate to the NP surface, is given in our previous work. ${ }^{[\mathrm{S} 4]}$ In the following, the chemistry of DOX referring to the production phase of the trajectories TRAJ 1 and TRAJ 2 is discussed. Table S2 provides a statistical evaluation of the distances of the respective centers of mass (COMs) of the $\mathrm{Au}_{114}$ core, the anthracycline fragment of DOX, and the indole residue of W5 as well as the number of contacts between DOX and the caps of the Au-NP for the whole production part of TRAJ 1, compiling separate estimates for the observed adsorption configurations.

Table S2. Average values and standard deviations of the distances W5-DOX, Au $114-\mathrm{DOX}$, and W5-Au114, and of the number of contacts between the methyl thiolate caps and DOX; separate estimates are compiled for all observed adsorption configurations within TRAJ 1. The letter in brackets refers to the displayed structure in Figure 1 of the main text.

\begin{tabular}{|l|c|c|c|c|}
\hline \multicolumn{1}{|c|}{ Time $\boldsymbol{t} / \mathbf{n s}$} & $\mathbf{0}<\boldsymbol{t}<\mathbf{1 5}$ & $\mathbf{1 5}<\boldsymbol{t}<\mathbf{1 4 0}$ & $\mathbf{1 4 0}<\boldsymbol{t}<\mathbf{1 7 5}$ & $\mathbf{1 7 5}<\boldsymbol{t}<\mathbf{2 5 0}$ \\
\hline Adsorption configuration & W5-DOX & CAPS-DOX- & W5-DOX -- & $\begin{array}{c}\text { DOX non- } \\
\text { specifically (C) }\end{array}$ \\
& $(\mathbf{A})$ & W5 (E) & CAPS (D) & $0.51 \pm 0.15$ \\
Distance $(\mathrm{W} 5-\mathrm{DOX}) / \mathrm{nm}$ & $0.37 \pm 0.04$ & $0.61 \pm 0.10$ & $0.38 \pm 0.08$ & $1.62 \pm 0.37$ \\
Distance $\left(\mathrm{Au}_{114-\mathrm{DOX}) / \mathrm{nm}}\right.$ & $2.15 \pm 0.23$ & $1.58 \pm 0.18$ & $1.67 \pm 0.20$ & $1.51 \pm 0.14$ \\
Distance $\left(\mathrm{W} 5-\mathrm{Au}_{114}\right) / \mathrm{nm}$ & $1.92 \pm 0.17$ & $2.06 \pm 0.09$ & $1.58 \pm 0.13$ & $328 \pm 111$ \\
Number of Contacts & $43 \pm 61$ & $352 \pm 145$ & $197 \pm 128$ & \\
CAPS-DOX & & & & \\
\hline
\end{tabular}


In the initial structure of the MD simulations, DOX is stacked by the tryptophan residue W5, corresponding to the most populated cluster of the DBP-DOX conjugate at $310 \mathrm{~K} .{ }^{[\mathrm{S} 7]}$ Already during relaxation of the system (Figure S4), the drug is attracted by the methyl thiolate caps of the Au-NP and moves toward the surface of the Au-NP but still interacts strongly with W5. While in the initial period of the production phase ( $t<15 \mathrm{ns)}$ DOX is stacked by W5 (corresponding to adsorption state A in Figure 1 of the main text), the CAPS-DOX-W5 sandwich (corresponding to adsorption state $\mathbf{E}$ in Figure $\mathbf{1}$ of the main text) is formed in the time range $15 \mathrm{~ns}<t<140 \mathrm{~ns}$. The switch in adsorption configuration is accompanied by an increased or decreased DOX-W5 and $\mathrm{Au}_{114}-\mathrm{DOX}$ distance, respectively (Table S2). Besides, a significantly higher number of contacts of DOX with the caps is observed (Table S2). Thereafter, the drug switches back to the W5-DOX configuration, in which, however, DOX is oriented perpendicular to the NP surface (corresponding to adsorption state D in Figure 1 of the main text). Consequently, the DOX-W5 distance and the number of contacts between the drug and the caps are reduced (Table S2). At the end of the first trajectory ( $t>175 \mathrm{~ns}$ ), DOX leaves W5 and intercalates nonspecifically between the caps of the NP and the backbone of the peptide (corresponding to adsorption state $\mathbf{C}$ in Figure 1 of the main text). This results in a larger W5-DOX distance (Table S2), since both W5 and DOX, independent from each other, interact with the caps of the Au-NP. The latter is visible by the reduced W5-Au $\mathrm{Au}_{14}$ distance and the increasing number of contacts of DOX with the caps (Table S2). For statistical analyses of TRAJ 1 and further information beyond Table S2, the reader is directed to reference [S4].

Cluster analysis of TRAJ 1 reveals two, two, seven, and one representative structure(s) for the DOX-W5 (A), perpendicular DOX-W5 (D), CAPS-DOX-W5 sandwich (E), and the non-specific CAPS-DOX-peptide (C) configurations, respectively. The calculated stabilization energies for each of these representative structures are compiled in Figure 1 of the main text. A statistical analysis of the DFT-derived binding energies can be found in our previous work. ${ }^{[\mathbf{S 4}]}$

Table S3 provides a statistical evaluation of the distances of the respective centers of mass (COMs) of the $\mathrm{Au}_{114}$ core, the anthracycline fragment of DOX, and the indole residue of W5 as well as the number of contacts between DOX and the caps of the Au-NP for the whole production part of TRAJ 2, compiling separate estimates for the observed adsorption configurations.

Table S3. Average values and standard deviations of the distances W5-DOX, Au $114-\mathrm{DOX}$, W5-W13, and of the number of contacts between the methyl thiolate caps and DOX; separate estimates are compiled for all observed adsorption configurations within TRAJ 2. The letter in brackets refers to the displayed structure in Figure 1 or Figure 4 of the main text.

\begin{tabular}{|c|c|c|}
\hline Time $t / \mathrm{ns}$ & $0<t<50$ & $50<t<125$ \\
\hline Adsorption configuration & W5-DOX-W13 -- CAPS (F) & W5-DOX-W13 (B) \\
\hline Distance (W5-DOX) / nm & $0.45 \pm 0.18$ & $0.46 \pm 0.15$ \\
\hline Distance $\left(\mathrm{Au}_{114}-\mathrm{DOX}\right) / \mathrm{nm}$ & $1.90 \pm 0.15$ & $2.14 \pm 0.17$ \\
\hline Distance (W5-W13) / nm & $0.78 \pm 0.14$ & $0.79 \pm 0.12$ \\
\hline Number of Contacts (CAPS-DOX) & $326 \pm 148$ & $35 \pm 49$ \\
\hline
\end{tabular}

Albeit DOX reveals strong tendency to move toward the caps of the Au-NP when stacked by W5, a different behavior of the drug is observed in the second MD trajectory. During relaxation of the system, W13 shifts in-between the caps and the W5-DOX complex, which enables DOX to intercalate between these two tryptophan residues in proximity to the surface of the NP (adsorption state $\mathbf{F}$ in Figure 4 in the main text). This sandwich configuration, observed in the first $50 \mathrm{~ns}$ of TRAJ 2, is in competition with the intercalation of DOX between W5 and W13 aside the NP surface with a weak interaction between DOX and NP, as obtained in the last 75 ns of this trajectory (adsorption state B in Figure $\mathbf{1}$ in the main text). 
The change in adsorption configuration is particularly visible by the significantly reduced number of contacts between the drug and the caps of the Au-NP (Table S3), whereas the distances of the two tryptophan residues as well as W5 and DOX remain virtually constant (Table S3).

Cluster analysis of TRAJ 2 reveals eight representative structures for the intercalation of DOX inbetween the two tryptophan residues W5 and W13 (configurations B and F). The calculated stabilization energies for these configurations are specified in Figure 1 or Figure $\mathbf{4}$ of the main text. Further information on TRAJ 2 as well as statistical analyses of the data shown in Table $\mathbf{S 3}$ are given in our

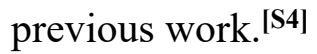

\section{Multi-scale modeling approach: volcano curves based on MD + DFT}

In this manuscript, we introduce a strategy to analyze a DDS component by multi-scale modeling, thereby combining MD simulations with DFT calculations. Figure S5 provides a sketch of our methodology, as discussed in the main text (Figure 2). We conceive that our screening approach is universal and not restricted to the investigated NP-DBP-DOX associate, but rather may be beneficial whenever detailed molecular insight into DDS components is pursued.

MD simulations:

Independent trajectories

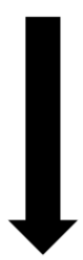

\section{Cluster representative} configurations

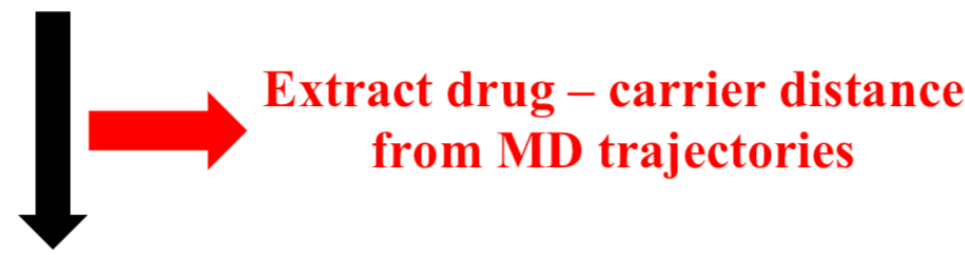

DFT calculations:

Quantify interaction energies

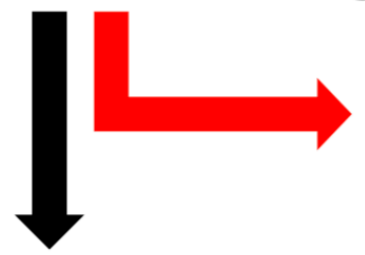

\section{Determine drug stabilization energy}

Plot stabilization energy as a

function of

drug - carrier distance:

volcano-shaped curve for

DDS interaction screening

Figure S5. Schematic representation of the combination of MD simulations and quantum chemical calculations, followed by specific data analysis, to assess the performance of a DDS component. 
The combination of MD simulations and DFT calculations, which has been introduced previously, ${ }^{[\mathbf{S 4}, \mathbf{S 7}, \mathbf{S 3 0}]}$ is not only a powerful combination for the assessment of the behavior of DDS components, but also is of importance to the electrocatalysis community. There, volcano plots are commonly constructed by the analysis of simple binding energies, derived from static DFT calculations, in which the influence of the adjacent electrolyte solution is often completely neglected or is described by implicit solvation models within the DFT code. ${ }^{[\mathbf{S 3 1}, 32]}$ Implicit solvation models, however, do not always provide accurate results, ${ }^{[\mathbf{S 3}]}$ calling for enhanced sampling techniques to properly account for the dynamics of the aqueous electrolyte solution at the solid/liquid interface. Ab initio molecular dynamics simulations have been established for an improved description of electrochemical solid/liquid interfaces

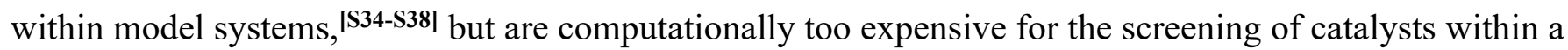
homologous series of materials. This suggests that it would be feasible to transfer the combined MD + DFT approach, as discussed on the example of the investigated DDS component (Figure S1), to the electrocatalysis community. A potential scenario to adopt the methodology of Figure S5 for the screening of electrocatalysts within a class of materials is illustrated in Figure S6.

\section{MD simulations: \\ Independent trajectories for all potential reaction intermediates suggested within the electrocatalytic cycle}

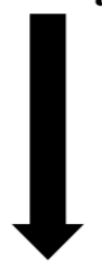

Cluster representative configurations of all reaction intermediates at the solid/liquid interface

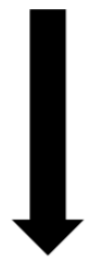

\section{QM/MM calculations to quantify free energies, enclosing the surrounding aqueous electrolyte}

\section{Derivation of linear scaling relationships from the calculated free energies}

Figure S6. Scheme of the multi-scale modeling approach, consisting of a combination of MD simulations and DFT (QM/MM) calculations, to derive linear scaling relationships within a class of materials. 
We believe that it would be very advantageous to compare the common procedure of deriving linear scaling relationships, based on static DFT calculations without surrounding solvent or implicit solvation models, to the suggested procedure in Figure S6. This may provide further insight into the real contribution of the solvent on the stabilization of reaction intermediates at the solid/liquid interface and clarify whether the usage of simple static DFT calculations is justified for the description of the dynamic multi-scale catalyst/electrolyte junction ${ }^{[\mathbf{S 3 9 ]}}$ within screening studies.

\section{High-throughput screening of drug-delivery system components}

The development of a multi-component DDS is computationally demanding. Therefore, based on the results for the NP-DBP-DOX conjugate discussed in the main text (Figure 4), we suggest (Figure S7) a high-throughput screening approach, a method of common interest within both the catalysis and biosimulation communities, ${ }^{[\mathbf{S 4 0 - S 4 3 ]}}$ for the design of DDS constituents.

\section{Component I: Biomolecule}

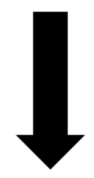

MD simulations: intercalation of the drug within the biomolecule observed?

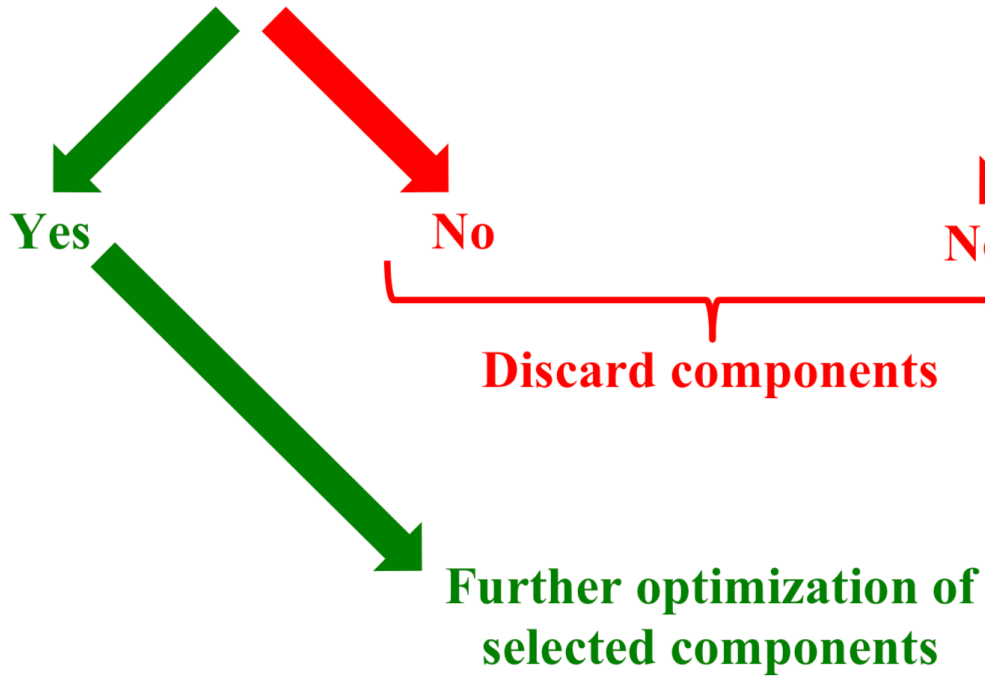

Component II:

Nanoparticle

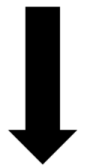

MD/DFT screening: interaction of the drug and NP surface attractive?

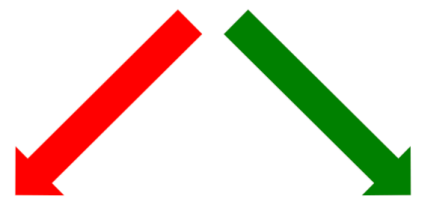

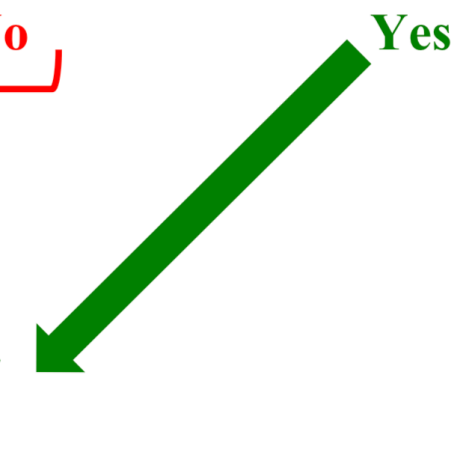

Figure S7. High-throughput screening for a three-component DDS, consisting of a drug, a biomolecule, and a nanoparticle (NP).

We assume that the DDS component consists of three constituents; that is, a drug and a supportive biomolecule as well as a carrier nanoparticle (NP). Following the insight gained from the NP-DBP-DOX conjugate in this work, we would select potential building blocks by independent screening of the biomolecule and NP. Relating to the biomolecule, we define the selection criterion that intercalation of the drug in-between the biomolecule residues is indispensable. ${ }^{[\mathbf{S 4}, \mathbf{S 8}]}$ Hence, all potential biomolecules that do not possess at least two appropriately spaced $\pi$-stacking residues (such as the tryptophan entities in the DBP, Figure S1) can be ruled out directly. Running MD trajectories aims at 
identifying biomolecules that intercalate the cytostatic agent in-between two $\pi$-stacking residues: only these biomolecules are further processed as potential constituents of a more complex DDS.

Performing single-point calculations for the drug in proximity to the NP, thereby adopting geometries obtained from a MD trajectory, facilitates quantifying the drug-NP interaction: only NP compositions, for which the interaction energy drug-NP is sufficiently attractive (such as encountered with the thiolateprotected Au-NP in our study, Figure 2 in the main text), are considered for subsequent optimization.

Finally, the identified biomolecules and NP configurations are investigated in conjunction with the drug, such as discussed in this manuscript on the example of the NP-DBP-DOX conjugate. Assume that $X$ biomolecules and $Y$ NPs are considered as potential building blocks of a three-component DDS; hence, the suitability of $X \times Y$ possible three-body constituents ought to be investigated. Following the above discussion, we select only biomolecules that meet the criteria of Figure S7. Thus, $X$ is disassembled into suitable $\left(x_{\mathrm{sel}}\right)$ and rejected $\left(x_{\mathrm{rej}}\right)$ compounds. The same strategy is conducted for the NP by decomposing $Y$ into suitable $\left(y_{\text {sel }}\right)$ and rejected $\left(y_{\text {rej }}\right)$ constituents. Consequently, $\left(X-x_{\text {rej }}\right) \times\left(Y-y_{\text {rej }}\right)=x_{\text {sel }} \cdot y_{\text {sel }}$ components are selected as potential building blocks of a prospective multi-component DDS, which are subject to further studies, including experiments.

\section{References:}

[S1] Lopez-Acevedo, O.; Akola, J.; Whetten, R.L.; Grönbeck, H.; Häkkinen, H. Structure and Bonding in the Ubiquitous Icosahedral Metallic Gold Cluster $\mathrm{Au}_{144}(\mathrm{SR})_{60 .}$. J. Phys. Chem. Lett. 2009, 113, 5035-5038.

[S2] Schaaff, T.G.; Shafigullin, M.N.; Khoury, J.T.; Vezmar, I.; Whetten, R.L. Properties of a Ubiquitous $29 \mathrm{kDa}$ Au:SR Cluster Compound. J. Phys. Chem. B 2001, 105, 8785-8796.

[S3] Scardi, P.; Billinge, S.J.L.; Neder, R.; Cervellino, A. Celebrating 100 years of the Debye scattering equation. Acta Crystal 2016, A72, 589-590.

[S4] Exner, K.S.; Ivanova, A. Identifying a gold nanoparticle as a proactive carrier for transport of a doxorubicin-peptide complex. Coll. Surf. B 2020, 194, 111155.

[S5] Dixit, S. B.; Bhasin, R.; Rajasekaran, E.; Jayaram, B. Solvation thermodynamics of amino acids Assessment of the electrostatic contribution and force-field dependence. Faraday Trans. 1997, 93, 1105-1113.

[S6] Canle, M.; Ramos, D. R.; Santaballa, J. A. A DFT study on the microscopic ionization of cysteine in water. Chem. Phys. Lett. 2006, 417, 28-33.

[S7] Gocheva, G.; Ilieva, N.; Peneva, K.; Ivanova, A. Characterization of the interaction forces in a drug carrier complex of doxorubicin with a drug-binding peptide. Chem. Biol. Drug Des. 2018, 91, 874-884.

[S8] Gocheva, G.; Peneva, K.; Ivanova, A. Self-assembly of doxorubicin and a drug-binding peptide studied by molecular dynamics. Chem. Phys. 2019, 525, 110380.

[S9] Duan, Y.; Wu, C.; Chowdhury, S.; Lee, M. C.; Xiong, G.; Zhang, W.; Yang, R.; Cieplak, P.; Luo, R.; Lee, T.; Caldwell, J.; Wang, J.; Kollman, P. A point-charge force field for molecular mechanics simulations of proteins based on condensed-phase quantum mechanical calculations. $J$. Comput. Chem. 2003, 24, 1999-2012.

[S10] Jorgensen, W. L.; Chandrasekhar, J.; Madura, J. D.; Impey, R. W.; Klein, M. L. Comparison of simple potential functions for simulating liquid water. J. Chem. Phys. 1983, 79, 926-935.

[S11] Jorgensen, W. L.; Madura, J. D. Temperature and size dependence for Monte Carlo simulations of TIP4P water. Mol. Phys. 1985, 56, 1381-1392.

[S12] Tsoneva, Y.; Tadjer, A.; Lelle, M.; Peneva, K.; Ivanova, A. Molecular Structure and Pronounced Conformational Flexibility of Doxorubicin in Free and Conjugated State within a Drug-Peptide Compound. J. Phys. Chem. B 2015, 119, 3001-3013. 
[S13] Giri, A.K.; Spohr, E. Conformational Equilibria of Organic Adsorbates on Nanostructures in Aqueous Solution: MD Simulations. J. Phys. Chem. C 2015, 119, 25566-25575.

[S14] Bayly, C. I.; Cieplak, P.; Cornell, W. D.; Kollman, P.A. A well-behaved electrostatic potential based method using charge restraints for deriving atomic charges: the RESP model. J. Phys. Chem. 1993, 97, 10269-10280.

[S15] Berendsen, H. J. C.; Postma, J. P. M.; van Gunsteren, W. F.; DiNola, A.; Haak, J. R. Molecular dynamics with coupling to an external bath. J. Chem. Phys. 1984, 81, 3684-3690.

[S16] Bussi, G.; Donadio, D.; Parrinello, M. Canonical sampling through velocity rescaling. J. Chem. Phys. 2007, 126, 014101-014107.

[S17] Ryckaert, J. P.; Ciccotti, G.; Berendsen, H. J. C. Numerical integration of the cartesian equations of motion of a system with constraints: molecular dynamics of n-alkanes. J. Comput. Phys. 1977, 23, 327-341.

[S18] Miyamoto, S.; Kollman, P.A.A. Settle: An analytical version of the SHAKE and RATTLE algorithm for rigid water models. J. Comput. Chem. 1992, 13, 952-962.

[S19] Darden, T.; York, D.; Pedersen, L. Particle mesh Ewald: An N $\log (\mathrm{N})$ method for Ewald sums in large systems. J. Chem. Phys. 1993, 98, 10089-10092.

[S20] Byrd, R. H.; Lu, P.; Nocedal, J. A limited memory algorithm for bound constrained optimization. SIAM J. Scientif. Statistic. Comput. 1995, 16, 1190-1208.

[S21] Zhu, C.; Byrd, R. H.; Nocedal, J. Algorithm 778: L-BFGS-B: Fortran subroutines for large-scale bound-constrained optimization. ACM Trans. Math. Softw. 1997, 23, 550-560.

[S22] Allen, M. P.; Tildesley, D. J. Computer Simulation of Liquids, Clarendon Press: Oxford, 1987.

[S23] Abraham, M. J.; Murtola, T.; Schulz, R.; Pálla, S.; Smith, J. C.; Hess, B.; Lindahl E. GROMACS: High performance molecular simulations through multi-level parallelism from laptops to supercomputers. SoftwareX 2015, 1-2, 19-25.

[S24] Humphrey, W.; Dalke, A.; Schulten, K. VMD: Visual molecular dynamics. J. Mol. Graph. 1996, 14, 33-38.

[S25] Jarvis, R. A.; Patrick, E. A. Clustering Using a Similarity Measure Based on Shared Near Neighbors. IEEE Trans. Comput. 1973, C22, 1025-1034.

[S26] Grimme, S.; Antony, J.; Ehrlich, S.; Krieg, S. A consistent and accurate ab initio parametrization of density functional dispersion correction (DFT-D) for the 94 elements H-Pu. J. Chem. Phys. 2010, 132, 154104.

[S27] Hay, P. J.; Wadt, W. R. Ab initio effective core potentials for molecular calculations. Potentials for K to Au including the outermost core orbitals. J. Chem. Phys. 1985, 82, 299-310.

[S28] Boys, S. F.; Bernardi, F. The calculation of small molecular interactions by the differences of separate total energies. Some procedures with reduced errors. Mol. Phys. 1970, 19, 553-566.

[S29] Frisch, M. J.; Trucks, G. W.; Schlegel, H. B.; Scuseria, G. E.; Robb, M. A.; Cheeseman, J. R.; Scalmani, G.; Barone, V.; Petersson, G. A.; Nakatsuji, H.; Li, X.; Caricato, M.; Marenich, A. V.; Bloino, J.; Janesko, B. G.; Gomperts, R.; Mennucci, B.; Hratchian, H. P.; Ortiz, J. V.; Izmaylov, A. F.; Sonnenberg, J. L.; Williams-Young, D.; Ding, F.; Lipparini, F.; Egidi, F.; Goings, J.; Peng, B.; Petrone, A.; Henderson, T.; Ranasinghe, D.; Zakrzewski, V. G.; Gao, J.; Rega, N.; Zheng, G.; Liang, W.; Hada, M.; Ehara, M.; Toyota, K.; Fukuda, R.; Hasegawa, J.; Ishida, M.; Nakajima, T.; Honda, Y.; Kitao, O.; Nakai, T. Vreven, K. Throssell, J. A. Montgomery, Jr., J. E. Peralta, F. Ogliaro, M. J. Bearpark, H.; Heyd, J. J.; Brothers, E. N.; Kudin, K. N.; Staroverov, V. N.; Keith, T. A.; Kobayashi, R.; Normand, J.; Raghavachari, K.; Rendell, A. P.; Burant, J. C.; Iyengar, S. S.; Tomasi, J.; Cossi, M.; Millam, J. M.; Klene, M.; Adamo, C.; Cammi, R.; Ochterski, J. W.; Martin, R. L.; Morokuma, K.; Farkas, O.; Foresman, J. B.; Fox, D. J. Gaussian 16, Revision B.01, Gaussian, Inc., Wallingford CT, 2016.

[S30] Petrova, J.; Gocheva, G.; Ivanova, N.; Iliev, S.; Atanasova, B.; Madjarova, G.; Ivanova, A. Molecular simulation of the structure of folate and antifolates at physiological conditions. J. Mol. Graph 2019, 87, 172-184.

[S31] Halck, N. B.; Petrykin, V.; Krtil, P.; Rossmeisl, J. Beyond the Volcano Limitations in Electrocatalysis - Oxygen Evolution Reaction. Phys. Chem. Chem. Phys. 2014, 16, 13682-13688. 
[S32] Exner, K.S.; Anton, J.; Jacob, T.; Over, H. Controlling Selectivity in the Chlorine Evolution Reaction over $\mathrm{RuO}_{2}$-Based Catalysts. Angew. Chem. Int. Ed. 2014, 53, 11032-11035.

[S33] Hörmann, N.G.; Andreussi, O.; Mazari, N. Grand canonical simulations of electrochemical interfaces in implicit solvation models. J. Chem. Phys. 2019, 150, 041730.

[S34] Cheng, J.; Sprik, M. Alignment of electronic energy levels at electrochemical interfaces. Phys. Chem. Chem. Phys. 2012, 14, 11245-11267.

[S35] Hansen, M.H.; Rossmeisl, J. pH in Grand Canonical Statistics of an Electrochemical Interface. $J$. Phys. Chem. C 2016, 120, 29135-29143.

[S36] Hansen, M.H.; Nilsson, A.; Rossmeisl, J. Modelling $\mathrm{pH}$ and potential in dynamic structures of the water/Pt(111) interface on the atomic scale. Phys. Chem. Chem. Phys. 2017, 19, 23505-23514.

[S37] Yang, X.H.; Cuesta, A.; Cheng, J. Computational Ag/AgCl Reference Electrode from Density Functional Theory-Based Molecular Dynamics. J. Phys. Chem. B 2019, 123, 10224-10232.

[S38] Le, J.-B.; Cheng, J. Modeling electrochemical interfaces from ab initio molecular dynamics: Water adsorption on metal surfaces at potential of zero charge. Curr. Opin. Electrochem. 2020, 19, 129-136.

[S39] Mueller, J.; Fantauzzi, D.; Jacob, T. Multiscale Modeling of Electrochemical Systems. In: Electrocatalysis, Volume 14 (Eds.: Alkire, R.C.; Kolb, D.M.; Kibler, L.A.; Lipkowski, J.). WileyVCH, Weinheim, 2013.

[S40] Broach, J.R.; Thorner, J. High-throughput screening for drug discovery. Nature 1996, 384, 14-16.

[S41] Wahler, D. Reymond, J.-L. High-throughput screening for biocatalysts. Curr. Opin. Biotech 2001, 12, 535-544.

[S42] Greeley, J.; Jaramillo, T.F.; Bonde, J.; Chorkendorff, I.; Norskov, J.K. Computational highthroughput screening of electrocatalytic materials for hydrogen evolution. Nature Mater. 2006, 5 , 909-913.

[S43] Exner, K.S. Universality in Oxygen Evolution Electrocatalysis: High-Throughput Screening and a Priori Determination of the Rate-Determining Reaction Step. ChemCatChem 2020, 12, 2000-2003. 